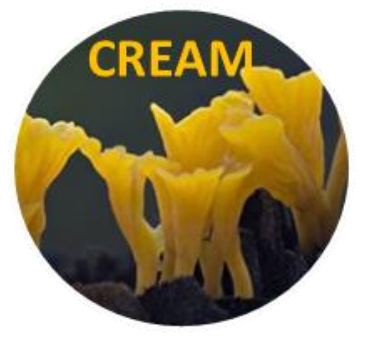

\title{
Molecular characterization of wood ear mushrooms [Auricularia sp.] from Kakamega Forest in Western Kenya
}

\author{
Onyango $\mathrm{BO}^{1^{*}}$, Mbaluto $\mathrm{CM}^{2}$, Mutuku $\mathrm{CS}^{1}$ and Otieno $\mathrm{DO}^{3}$ \\ ${ }^{1}$ Department of Biological Sciences, Chuka University, P. O. Box 109-40600, Chuka, Kenya \\ ${ }^{2}$ Kenyatta University, P.O. Box 43844-00100. Nairobi, Kenya \\ ${ }^{3}$ Kenya Industrial Research and Development Institute, P.O.BOX 30650-00100 Nairobi, Kenya
}

Onyango BO, Mbaluto CM, Mutuku CS, Otieno DO 2016 - Molecular characterization of wood ear mushrooms [Auricularia sp.] from Kakamega Forest in Western Kenya. Current Research in Environmental \& Applied Mycology 6(1), 51-60, Doi 10.5943/cream/6/1/6

\begin{abstract}
Mushrooms of the genus Auricularia, generally termed wood ear mushrooms are in high demand in Western Kenya due to their numerous medicinal and nutritional properties. Interventions to characterize and conserve the native wood ear mushrooms are necessary to mitigate possible extinction of this valuable bio-resource. Currently, the species richness and bio-geographical relatedness of the Kenyan native wood ears is not fully elucidated. This study used molecular sequence analysis of the internal transcribed spacer (ITS) and the 28S nuclear ribosomal large subunit (nLSU) genes in species delimitation of six strains of wood ear mushrooms native to Kakamega Forest. Phylogeny of both the ITS and nLSU gene regions showed that three strains clustered with Auricularia delicata while the other three strains clustered with Auricularia polytricha at bootstrap support values of above 97\%. An intragenomic dichotomy appeared to occur in the Auricularia delicata strains based on the genetic distance of the nLSU gene sequences. The wood ear mushrooms identified from the Kakamega Forest strains were Auricularia delicata and Auricularia polytricha and not Auricularia auricula as previously reported. This rich biodiversity needs further exploration to widen the nutritional and medicinal base of the rural populace who depend on the mushrooms through conservation, cultivation and commercialization activities.
\end{abstract}

Key words - Mushrooms - morphology - phylogeny - taxonomy

\section{Introduction}

Research on edible fungi in the world has concentrated on a few species that are commercially cultivated (FAO 2015). The consequence of this is that the biology and ecology of some edible species collected from the wild, particularly in developing countries are poorly known. In Western Kenya, mushrooms of the genus Auricularia, generally termed wood ear mushrooms are in high demand due to their numerous medicinal and nutritional properties (Palapala 2006). Communities residing around Kakamega Forest indiscriminately harvest the wood ear mushrooms for food and medicine, which threatens to deplete this valuable bio-resource (Onyango et al. 2011). Interventions to characterize and conserve these native mushrooms are necessary to mitigate possible extinction due overharvesting and the rapid destruction of its forest habitat. Taxonomic characterization of the wood ear mushroom will enhance its commercialization potential and provide information for its utilization and management. 
EXTERNAL BASIDIOME
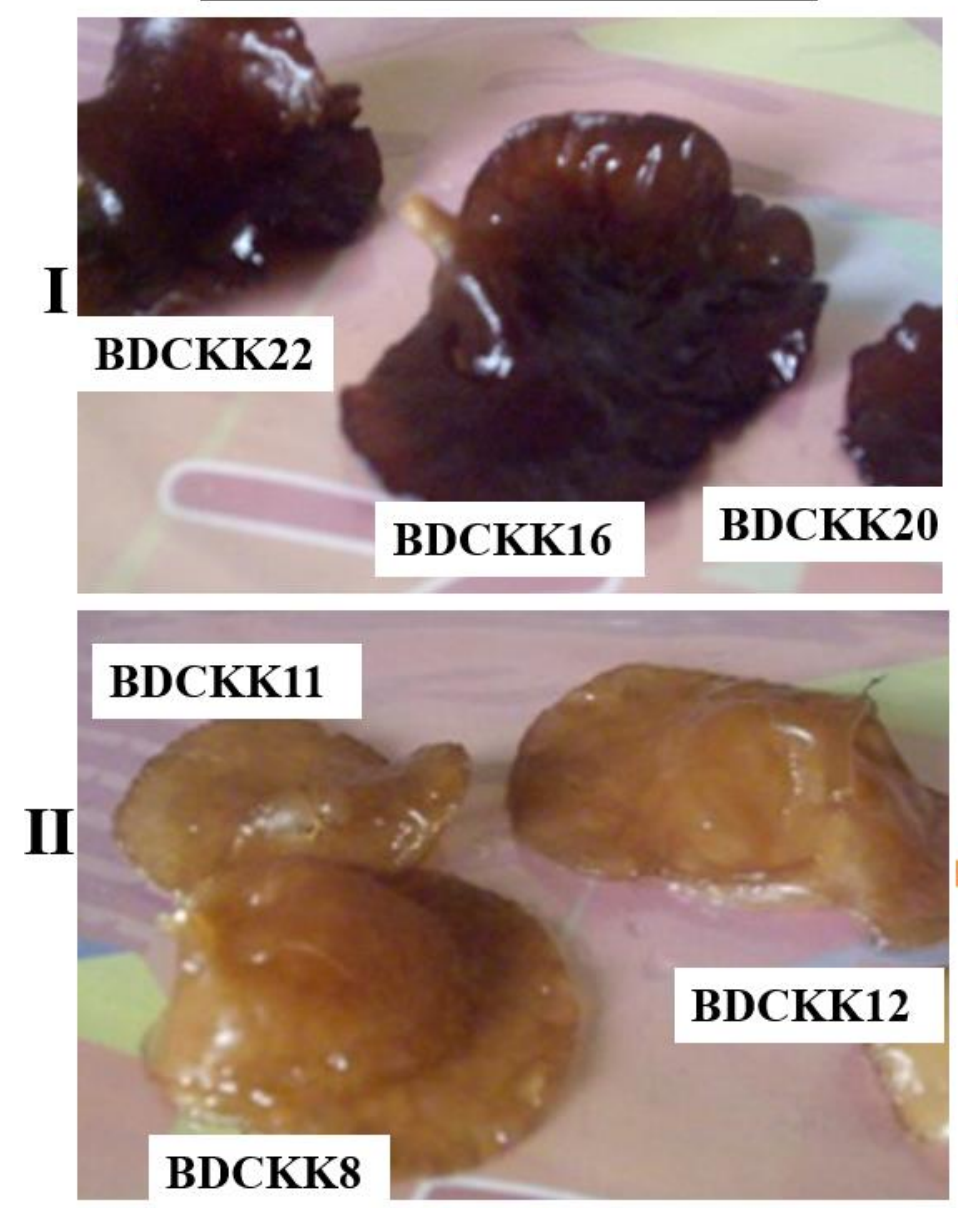

\section{INTERNAL BASIDIOME}
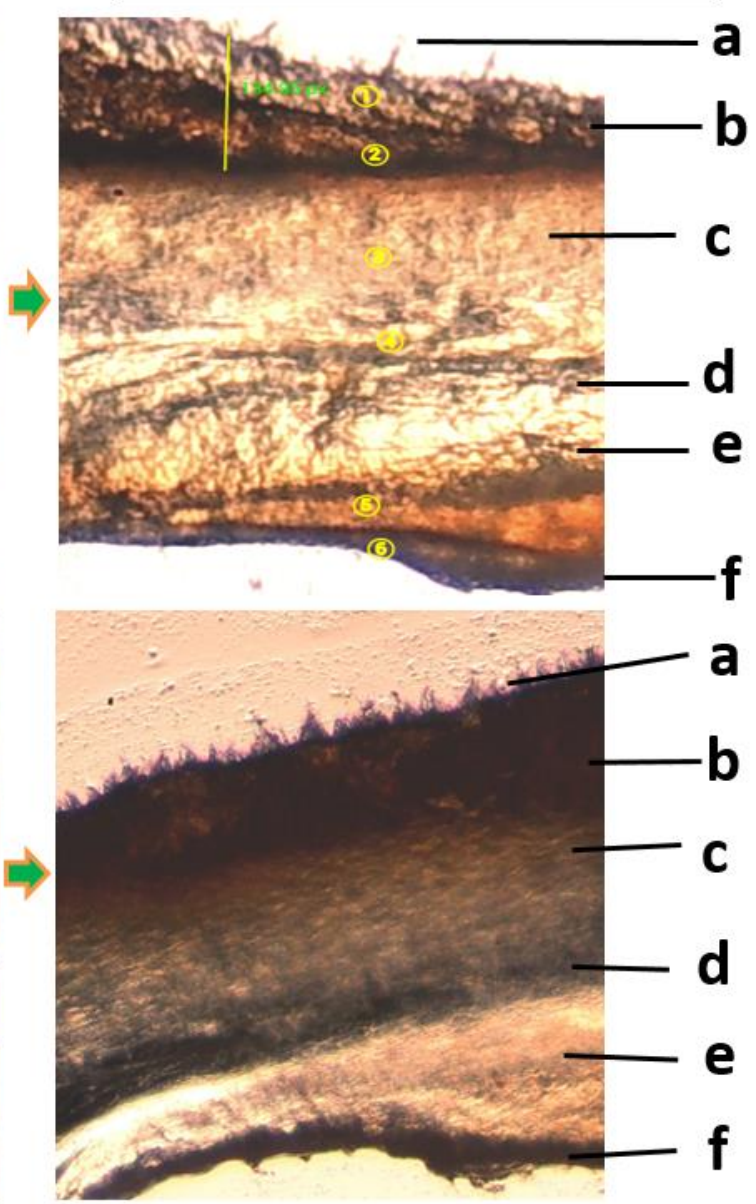

Fig. 1 - External morphology of wood ear basidiomes collected from Kakamega Forest and the cross sections showing hyphal stratification and abhymenial hairs. The scale of the cross sections is $134 \mu \mathrm{m}$. Group I - Black strains, Group II - Brown strains. Note the prominent stipe on sample BDCKK16. a. Abhymenial hairs b. Zona compacta c. Zona laxa superioris d. Medulla e. Zona laxa inferioris $\mathrm{f}$. Hymenium

Macro and micro-morphological characterization of the genus Auricularia has proven inconclusive in species delineation due to numerous convergent morphologies (Martin et al. 2004, Tang et al. 2010). For instance, macroscopic features of Auricularia vary with age of the specimen, exposure to light, availability of moisture and other environmental factors (Bandara et al. 2015). The taxonomic scheme of Auricularia has for a long period of time considered Lowy's monograph (Lowy 1951) which emphasised a strongly or weakly differentiated medulla in the internal basidiome structure. In recent times however, a new taxonomic character within the internal basidiome called a schizomedulla led to re-classification of two novel species; Auricularia subglabra and Auricularia scissa (Looney et al. 2013). Such morphological plasticity and absence of clearly distinguishable features has limited adequate discrimination of the genus Auricularia with clear genetic lineages. Onyango et al. (2011) analysed Auricularia mushrooms of Kakamega Forest using external and internal features of the basidiome and indicate that $A$. auricularia species are abundant in the Forest. However, a low dissimilarity index of 0.318 was observed indicating the limitation of morphological markers in determining genetic diversity. Thus, the species richness and bio-geographical relatedness of the Kenyan native wood ears is not fully elucidated. A diagnostic diversity study using more accurate molecular markers is therefore necessary. 
Table 1 Accession numbers and countries of origin of sequences used for phylogenetic analyses. Sequences newly produced in this study are shown in bold.

\begin{tabular}{llll}
\hline Identity of strain & ITS gene & nLSU gene & Country \\
\hline Auricularia polytricha BDCKK16 & KR605640 & KR605636 & Kenya \\
Auricularia polytricha BDCKK22 & KR605642 & KR605638 & Kenya \\
Auricularia polytricha BDCKK20 & KR605643 & KR605641 & Kenya \\
Auricularia delicata BDCKK8 & KR605639 & KR605644 & Kenya \\
Auricularia delicata BDCKK11 & KR605637 & KR605645 & Kenya \\
Auricularia delicata BDCKK12 & KR605645 & KR605646 & Kenya \\
Auricularia polytricha AP3/ AP910 & KF297984 & KM267736 & China \\
Auricularia polytricha AP10158/HN4076 & KF297985 & KF298021 & China/US \\
Auricularia polytricha M E7069/JMH43 & AJ537388 & KF298017 & Australia/Tanzania \\
Auricularia delicata HNSD & KF297965 & KF298000 & China \\
Auricularia delicata GIM5.424 & KF297964 & KF297999 & China \\
Auricularia delicata GIM5.177 & KF297963 & KF297998 & China \\
Auricularia fuscosuccinea TFB3463/PR1496 & JX065148 & KM396828 & US/China \\
Auricularia fuscosuccinea PR 1378 & AF291270 & KM396827 & China \\
Auricularia fuscosuccinea TFB11532 & JX065150 & KF298002 & US/China \\
Auricularia auricula-judae AFTOL-ID 1681 & DQ520099 & DQ520099 & Germany \\
Auricularia auricula-judae TFB4296/MW 447 & JX065174 & FJ644518 & US/Gemany \\
Auricularia auricula-judae Dai 13210 & KM396769 & KM396824 & China \\
Auricularia nigricans E3 & KP942816 & - & Nigeria \\
Auricularia nigricans TENN 056825 & NR_120153 & - & US \\
Auricularia nigricans TFB11410 & JX065176 & - & US \\
Exidia recisa & KF297985 & - & Germany \\
Ramaria rubella & - & JX287493 & United States \\
\hline
\end{tabular}

Molecular characterization of fungal genera provides more reliable taxonomic information; which is not influenced by environmental factors (Bandara et al. 2015). Markers such as Random Amplified Polymorphic DNA (RAPD), Restriction Fragment Length Amplified Polymorphism (RFLP) and Sequence Related Amplified Polymorphism (SRAP) have revolutionized fungal taxonomy (Park et $a l ., 2014)$. Potential barcodes targeted by these markers include the nuclear and ribosomal DNA (rDNA) cistrons. Sequence analysis of the (ITS) gene region of the rDNA has been proposed as the primary barcode of fungi (Schoch et al. 2012). The ITS gene region consists of ITS1-5.8S-ITS2; with the 5.8S rDNA gene being highly conserved and is therefore not very informative at the specie level of the taxonomy of fungi (Sharma et al., 2015). The intervening ITS1 and ITS2 regions show variation between fungal species with different hypervariable domains and high probabilities of successful intra and inter-specific variation (Korabecna et al. 2003). Due to these qualities, the two ITS gene regions can possibly be used to delimit closely related genera by a measure of their genetic distances (Martin et al. 2004).

The $28 \mathrm{~S}$ nLSU gene has also proven to be a useful marker in species delimitation. In addition to being easy to amplify, sequence and align, the nLSU gene has been shown to improve phylogenetic resolution (Park et al. 2014). The nLSU gene can be used alone or in combination with the ITS region to discriminate fungal species (Vellinga 2004). Therefore, inter-specific variations of ITS and nLSU gene sequences is a crucial step in construction of the phylogeny of native wood ear mushrooms of Kakamega Forest as a prerequisite for their conservation and domestication.

\section{Materials and Methods}

\section{Sources of germplasm}

Wood ear mushroom basidomes were obtained from Isecheno forest reserve of Kakamega Forest in Western Kenya between January and April 2013. The forest reserve is located between longitudes of $34^{\circ} 32^{\prime} 0^{\prime \prime} \mathrm{E}, 34^{\circ} 57^{\prime} 0^{\prime \prime} \mathrm{W}$ and latitudes of $0^{\circ} 7^{\prime} 30^{\prime \prime} \mathrm{N}, 0^{\circ} 10^{\prime} 15^{\prime \prime} \mathrm{S}$ (Wambua 2004). Sixteen fully mature basidiomes were excised from their fallen and decaying wood habitats using a sharp knife and put in polythene zip-lock bags. Care was taken to ensure the collected samples were at different 
stages of growth for proper representation. Diseased parts were avoided to eliminate contamination. Sixteen fruiting bodies were collected and assigned accession numbers then transferred to the laboratory for characterization.

\section{Morphological characterization}

Morphological analysis was done to determine differences between the external and internal features of the collected wood ear mushrooms based on Looney's monograph (Looney et al. 2013). External characters such as colour, shape, and stipe presence were evaluated by eye observation. Based on these characters, the samples were classified into two Groups I and II for further characterization. Six samples representative of the two groups with accession numbers BDCKK16, BDCKK22, BDCKK20, BDCKK8, BDCKK11 and BDCKK12 were selected for further morphological and molecular analysis. Thin cross sections of the basidiomes were obtained using sterile scalpel blades and immersed in a diluted solution of methyl blue stain for 10 minutes. The sections were mounted on $5 \% \mathrm{KOH}$ and viewed using a Nikon Eclipse 80i light microscope. Cross sections were photographed at $\times 4$ magnification for comparative analysis of the internal basidiome structures.

\section{DNA extraction}

Total genomic DNA was extracted from dried fruiting bodies of the selected six wood ear mushroom accessions by the Cetyl trimethyl-ammonium bromide (CTAB) method (White et al. 1990) with some modifications. Approximately $5 \mathrm{~g}$ was sliced from each fruiting body using a sterile scalpel blade and transferred into $1.2 \mathrm{ml}$ stripe tubes containing a stainless steel grinding balls. Strip tubes were cooled by immersing in liquid nitrogen previously stored at $-4^{\circ} \mathrm{C}$. Cooled mycelium was subsequently ground into fine powder using Geno grinder-2010 at 1750 strokes $\min ^{-1}$ for $5 \mathrm{~min}$. The samples were then centrifuged at $2000 \mathrm{rpm}$ for $1 \mathrm{~min}$ and the resultant powder at bottom of strip tubes was collected. Seven hundred micro-litres of CTAB extraction buffer was pre-warmed at $65^{\circ} \mathrm{C}$ for 1 min then $200 \mu \mathrm{L}$ of $2 \%$ polyvinylpyrrolidone was added to the samples and homogenized by gently inverting the tubes. The samples were incubated in a gently roaking water bath at $65^{\circ} \mathrm{C}$ for $1 \mathrm{~h}$. The tubes were removed and allowed to cool at room temperature, followed by addition of $700 \mu \mathrm{L}$ Chloroform Isoamyl Alcohol (24:1). The contents were mixed by gently inverting the tubes 10 times. Centrifugation followed at $4000 \mathrm{rpm}$ for $20 \mathrm{~min}$ and the aqueous phase transferred into freshly labelled strip tubes. Thereafter, $600 \mu 1$ of ice cold $40 \%$ isopropanol was added and incubated in a freezer at $20^{\circ} \mathrm{C}$ overnight. DNA was pelleted by centrifugation at $4000 \mathrm{rpm}$ for $20 \mathrm{~min}$ and then Ethano: Sodium acetate $(25: 1)$ was added and the mixture incubated at $-20^{\circ} \mathrm{C}$ for $45 \mathrm{~min}$. The supernatant was removed and the DNA washed twice with $500 \mu \mathrm{L}$ of $70 \%$ ethanol. The DNA was left to air dry for about 15 min and re-suspended in $100 \mu \mathrm{L} \mathrm{TE}_{0.1}$. Two microlitres of RNAse solution was added to each tube and incubated at $37{ }^{\circ} \mathrm{C}$ for $30 \mathrm{~min}$. The concentration and purity of DNA was estimated using a Nanodrop ${ }^{\mathrm{TM}}$ Lite Spectrophotometer (Thermo-Scientific Inc, USA) at 260-280 $\mathrm{nm}$. Horizontal gel electrophoresis (Thistle Scientific Ltd, USA) was done on a $0.8 \%$ (w/v) agarose gel at $100 \mathrm{~V}$ for 30 min and visualized under UV after staining with $2 \mu \mathrm{L}$ of GelRed ${ }^{\mathrm{TM}}$ (Thermo-Scientific, USA).

\section{Molecular characterization}

Polymerase chain reaction (PCR) amplification of the ITS region and $28 \mathrm{~S}$ rDNA gene was performed in a programmable Mastercycler thermocycler (C1000-BioRad, USA) using the PCR conditions described by Vellinga et al., (2003). The primers used for the ITS region were adopted from White et al. (1990) and included; forward primer ITS5 and the reverse primer ITS4. The primers used to amplify the nLSU region were derived from Gardes \& Bruns (1993) and included; forward primer LROR which aligns in the domain 26-42 and the reverse primer LR16 which aligns in the domain 1081-1065. The amplified products were separated by horizontal gel electrophoresis on $1.5 \%(\mathrm{w} / \mathrm{v})$ agarose gel on $0.5 \mathrm{X}$ TBE at $70 \mathrm{~V}$ for $60 \mathrm{~min}$ and visualized under UV after staining with $2 \mu 1 \mathrm{GelRed}^{\mathrm{TM}}$ (Thermo Scientific). The PCR amplicons were purified using a Thermo Scientific ${ }^{\circledR}$ GeneJET Purification Kit (EU, Lithuania) according to the manufacturer's specifications. 


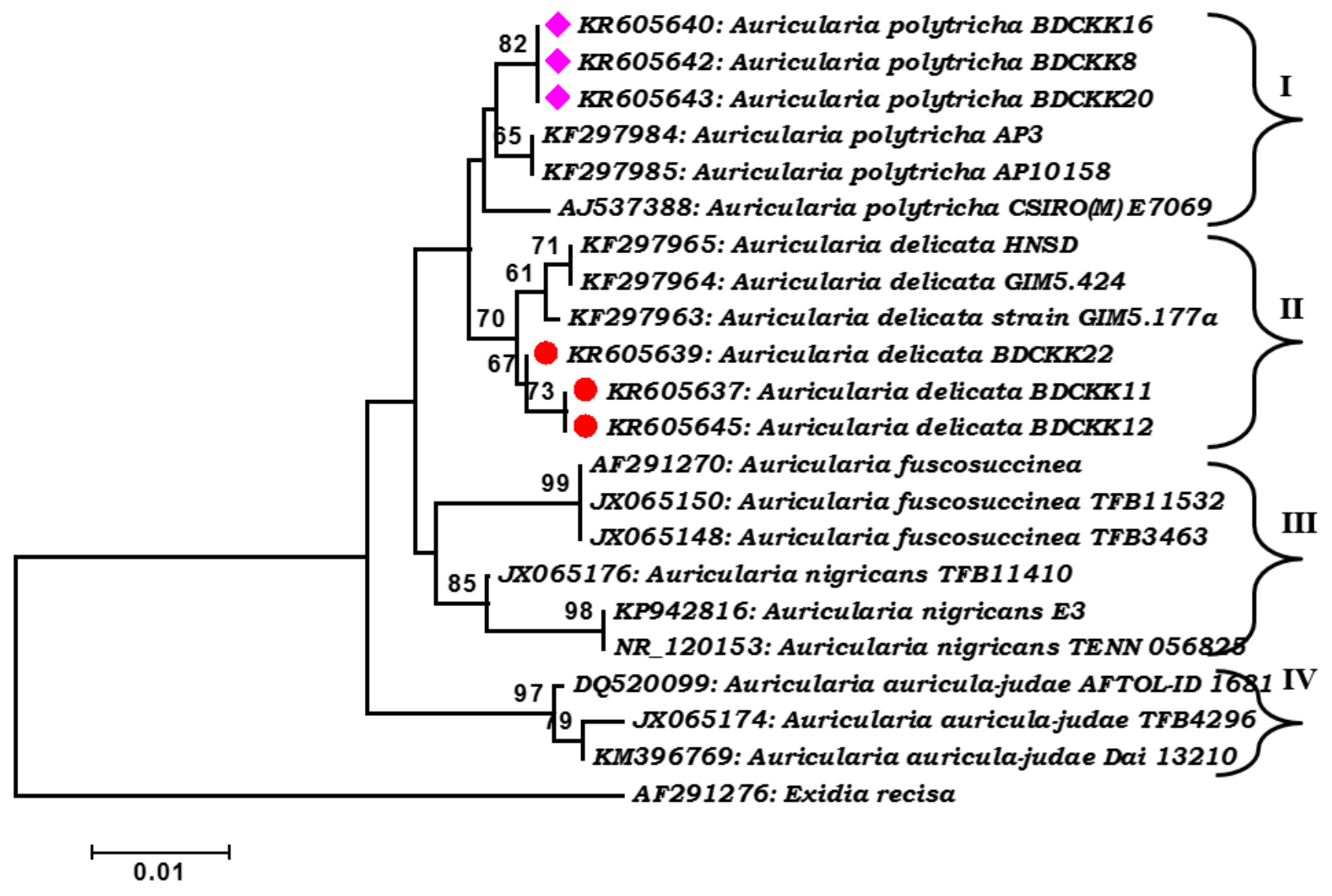

Fig. 2 - A Neighbour-Joining phylogenetic tree showing evolutionary relationships of the ITS gene sequences. The strains from this study are shown in different colours. The tree was constructed based on evolutionary distances computed using the Maximum-Composite Likelihood Method. The optimal tree with the sum of branch length $=0.10599626$ is shown. The tree is drawn to scale and the percentage of replicate trees in which the associated taxa clustered together in the bootstrap test (1000 replicates) are shown next to the branches (if $>50 \%$ ).

\section{Sequencing and data analysis}

The purified PCR products were pipetted into $10 \mu \mathrm{l}$ Epperndoff tubes and submitted to the Segolip Sequencing Unit, BecA-ILRI Hub, for capillary sequencing on a 3730xl DNA Analyzer (Thermo Fisher Scientific Inc. USA). Forward and reverse sequences were assembled and trimmed on CLC Main Workbench (CLC Bio Version 6.8.3). Assembled sequences were transferred to MEGA Version 6.0 and aligned using CLUSTAL W according to Tamura et al. (2011). Individual consensus sequences of the ITS and nLSU gene regions were used to evaluate closely related sequences at the NCBI GenBank (www.ncbi.blast.lnm.nih.gov) using Basic Local Alignment Tool (BLAST Query).

Nine sequences that recorded maximum identity hits of greater than $97 \%$ similarity to both the ITS and nLSU gene regions on the BLAST query were retrieved and used to supplement construction of phylogenetic trees on MEGA6.0. Three sequences of Auricularia auricula-judae for both the ITS and nLSU gene regions were also retrieved and used in the comparative phylogenetic analysis. Evolutionary histories were inferred using the Neighbour-Joining method and distances computed using the Maximum Composite Likelihood (Tamura \& Kumar, 2004). The percentage of replicate trees in which the associated taxa clustered together in the bootstrap test of 1000 replicates were shown next to the branches if the values were greater than $50 \%$. The outgroup used for the ITS and nLSU sequences phylogenetic analysis included Exidia recisa (Ditmar) Fr. and Ramaria ruballa (Schaeff.) R. H. Petersen, which are closely related species but outside the in-group of the Auriculariales (Weiß \& Oberwinkler 2001). 


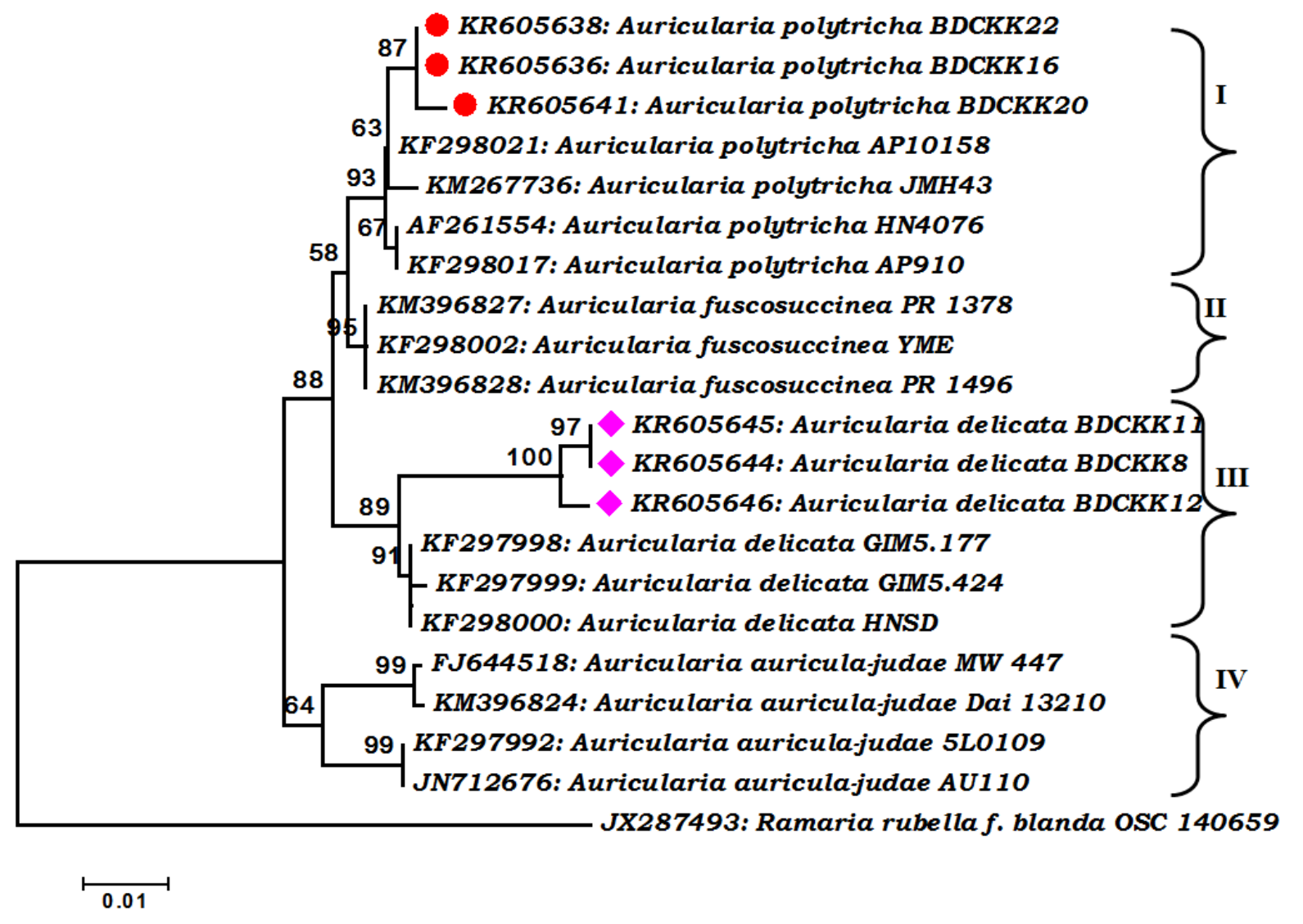

Fig. 3 - A neighbour-joining phylogenetic tree showing evolutionary relationship of the nLSU gene. The strains from this study are shown in different colours. The tree was constructed based on evolutionary distances computed using the Maximum-Composite Likelihood Method. The optimal tree with the sum of branch length $=0.18969980$ is shown. The tree is drawn to scale and the percentage of replicate trees in which the associated taxa clustered together in the bootstrap test (1000 replicates) are shown next to the branches (if $>50 \%$ ).

\section{Results}

\section{Morphological identification}

Morphological features of the wood ear mushrooms and the distinction of their internal basidiome structures are shown on Plate 1. Two groups I and II were identified with strains BDCKK16, BDCKK22 and BDCKK20 occurring in Group I. All Group I strains had long visible stipes and were black in colour. Strains BDCKK8, BDCKK11 and BDCKK12 were classified into Group II and were brown in colour with much shorter stipes. Variation within the internal basidiome structure was such that Group I strains had an average internal thickness of 0.8-1.2 mm. Other features of Group I strains were; abundant and gregarious abhymenial hairs - bearing separately visible individual hairs $(5-10 \mu \mathrm{m})$, a thick zona compacta layer $(21-40 \mu \mathrm{m})$ with an identifiable zona subcompacta superioris/inferioris, a large oblong zona laxa superioris (60-192 $\mu \mathrm{m})$, a tiny medulla (18-52 $\mu \mathrm{m})$, a narrower zona laxa inferioris $(42-120 \mu \mathrm{m})$ and a hymenium. Internal features of Group II strains were similar to those of Group I except in the nature of the abhymenial hairs and the size of the medulla. The medulla $(32-84 \mu \mathrm{m})$ was clearly visible and was surrounded by a larger zona laxa superioris $(72-201 \mu \mathrm{m})$ and a much narrower zona laxa inferioris $(15-35 \mu \mathrm{m})$. The abhymenial hairs were dense indistinguishable tufts of about $0.8-2.5 \mu \mathrm{m}$ in length. The zona compacta was quite thin $(15-27 \mu \mathrm{m})$ and it was not differentiated into a zona subcompacta superioris and a zona subcompacta inferioris. 


\section{Molecular analysis}

The strain identities, accession numbers and country of origin of the GenBank ITS and nLSU gene sequences of Auricularia mushroom species which were closely related to the six strains from Kakamega Forest are listed on Table 1. Previous reports on morphological characterization indicated that the wood ear mushroom species Auricularia auricula were abundant in Kakamega Forest. Based on this report, three sequences of the ITS and the nLSU genes of Auricularia auricula obtained from the GenBank were included in the analysis and are displayed on Table 1.

\section{Phylogenetic analysis of the ITS gene region}

The ITS gene amplification resulted in 670 base pairs. The Maximum Composite Likelihood phylogenetic analysis was inferred by the Neighbour Joining tree shown on Fig. 2. Four distinct Clades I, II, III and IV were observed. The six samples from this study clustered within Clades I and II with bootstrap support values of $65 \%$ for Auricularia polytricha and $70 \%$ for Auricularia delicata. None of the Kakamega Forest wood ears clustered in Clades III and IV with GenBank sequences identified as Auricularia auricula, Auricularia nigricans and Auricularia fuscosuccinea indicating absence of these species amongst the samples analysed. Strains BDCKK16, BDCKK22 and BDCKK20 were monophylectic within Clade I clustering with Auricularia polytricha strains AP3, AP10158 and CSIRO(M) E7069 from the GenBank at bootstrap support values of 58\%. Clade II consisted of strains BDCKK8, BDCKK11 and BDCKK12 which clustered with GenBank sequences identified as Auricularia delicata strains GIM5.424, GIM5.177a and HNSD, although the two groups were polyphyletic.

\section{Phylogenetic analysis of the nLSU gene region}

The Neighbour Joining nLSU gene phylogenetic tree based on Maximum Composite Likelihood analysis is shown in Fig. 3. A topology similar to the ITS gene phylogenetic tree was observed with four distinct clades I, II, III, IV. Strains BDCKK22, BDCKK16 and BDCKK20 were monophylectic within Clade I clustering with Auricularia polytricha strains AP10158, JMH43 and HN4076 at bootstrap support values of 93\%. Clade II had GenBank sequences of species of Auricularia fuscosuccinea which had no representation within the samples from this study. Strains BDCKK8, BDCKK11 and BDCKK12 were identified as Auricularia delicata strains within Clade III clustering with GenBank sequences strains GIM5.177, GIM5.424 and HNSD. However, strains BDCKK8, BDCKK11 within Clade III appeared to be distantly related suggesting a separate delineation. Clade IV consisted of Auricularia auricula-judae strains which were not represented within the samples obtained from Kakamega Forest.

\section{Discussion}

Domestication of edible mushrooms collected from the wild requires adequate taxonomic description. Proper taxonomic identification will enhance the management and commercialization of indigenous mushroom species and reduce the impact of excessive harvesting from their wild habitats. Accurate identification of wild edible mushrooms has the extension advantage of labelling of strains with correct designations, as they are introduced into new regions (Tang et al. 2010). Incorrect designation of strains may negatively impact on mushroom breeding programmes and interfere with intellectual property rights protection. Taxonomic identification is therefore an important step towards efficient utilization of mushroom germplasm collected from the wild.

Taxonomic studies of the genus Auricularia has been challenged by the absence of reliable morphological characters that represent a clear genetic diversity amongst its species (Looney et al. 2013). In this study, Groups I and II strains were initially categorized based on external basidiome colours, presence or absence of a stalk and the shape (Fig. 1). However this variation was not sufficient in distinguishing between the two groups as was observed by Bandara et al. (2015) in a study of a novel Auricularia sp. from Thailand that these traits overlap among different species. Similarly, the wood ear mushrooms of Kakamega Forest could not be adequately distinguished using internal basidiome structures. Both groups had a medulla region surrounded by two zones of loosely arranged 
hyphae known as Zona laxa superioris and Zona laxa inferioris. The medulla was however more prominent on Group II strains and smaller on Group I strains. The medulla is an important taxonomic variant in the Auricularia mushrooms and was used by Lowy (1952) to delimit the first ten species worldwide. Confounding observations in the presence/absence/size of the medulla in the Auricularia sp. have since been reported by Kirk et al. (2008) and Looney et al. (2013) which concur with the findings of this study. It was also evident that the nature of abhymenial hairs differed between Groups I and II strains, although Lowy et al. (1951) indicated that the hairs are not a sufficient criterion to allow a diagnosis of a species. These reports highlight the need for more morphological markers for identification of the genus Auricularia, or its combination with molecular markers. The DNA based molecular markers provide more accurate results in the speciation and cryptic diversity within the Basidiomycota (Milller \& Buyck 2002).

Molecular taxonomy and phylogeny potentially resolves the ambiguity generated from morphological plasticity. In this study, the overall topologies of the phylogenetic tree inferred by both the ITS (Fig. 2) and nLSU genes (Fig. 3) using Neighbour-Joining Maximum Composite Likelihood were similar in resolving the six strains from Kakamega Forest. Strains BDCKK8, BDCKK11 and BDCKK12 were identified as Auricularia polytricha while strains BDCKK22, BDCKK16 and BDCKK20 were identified as Auricularia delicata at above 97\% similarity values by BLAST analysis. Both the ITS and nLSU gene sequence analysis revealed very similar results in barcoding the Kakamega Forest strains. The minor differences observed in clade delination of some strains correlate well with the current species concepts (Schindell \& Miller 2005).

Analysis of the ITS and nLSU gene sequences revealed a wide evolutionary distance between Auricularia delicata strains from Kakamega Forest and those already deposited in the GenBank, signalling increased speciation of this group (Miller \& Buyck 2002). The GenBank samples closely related to the Kakamega Forest strains had solely been described in China, and had a long evolutionary distance between them (Fig. 2 and Fig. 3). Although the very first described Auricularia delicata species were from Africa (Bresadola et al. 1887), it was surprising that none of the strains from this study clustered with any of the African strains in the GenBank. These observations on the phylogeny of Auricularia delicata support two major conclusions. Firstly, the wide variation could be attributed to the possible occurrence of a novel group of Auricularia delicata amongst the Kakamega Forest strains that is yet to be described. Alternately, it may be due to the large number of Auricularia delicata sequences from China already deposited in the GenBank, which resulted in higher similarity indices with the Kakamega Forest strains than with sequences from other parts of the world.

Strains identified as Auricularia polytricha showed a broad geographic variation with GenBank sequences based on both the ITS and nLSU genes (Chen et al. 2001). Although Looney et al. (2013) reported that Auricularia polytricha is a synonym of Auricularia nigricans, the strains from this study appeared to form a separate dichotomy based on the ITS tree. The Kakamega Forest strains had maximum similarity indices with representative samples from China, Germany, United States and Tanzania (Table 1). This wide distribution of the Auricularia polytricha clade indicates its better adaptability to diverse ecological regions compared to the Auricularia delicata strains which had a lower distribution. For instance, the Tanzanian strain JMH43 obtained from Kizimzumwi Forest near Kisarawe (Hussein et al. 2014) had 100\% similarity index with strain BDCKK11 from Kakamega Forest. Kizimzumwi Forest is within the same geographic latitude as Kakamega Forest and has identical climatic conditions. At the same time, the indigenous tree species within the two regions are similar, yet Auricularia species are known to be highly selective to the host trees, which possibly explain the close identity of Kenyan and Tanzanian species. It is however necessary to further elucidate the evolutionary distribution of Auricularia polytricha strains closely related to the Kakamega Forest strains.

\section{Conclusions}

The first detailed study of the taxonomy of Auricularia mushrooms from Kakamega Forest used morphological markers and resulted in a dendogram of three clusters, suggesting three distinct species. Molecular analysis using the ITS and nLSU genes has however shown that there are two 
species; Auricularia delicata and Auricularia polytricha mushrooms in the forest. The ability of molecular markers to clearly delineate these species may also be extended to evaluate genetic variability within species with the possibility of identification of novel strains. An intra-genomic dichotomy appeared to occur in the Auricularia delicata strains based on the genetic distance of the nLSU gene sequences. Further exploration of the biodiversity of Kenyan wood ear mushrooms is necessary to examine their distribution and to identify type collections for herbarium preservation at the Kenya National Museums. This will increase scientific information on the existing wood ear species and also widen the nutritional and medicinal base of the rural populace who depend on the mushrooms, through conservation, cultivation and commercialization activities.

\section{Acknowledgement}

The authors are grateful to the Australian Government (AusAID) through a BecA-ILRI HubCSIRO initiative on Food and Nutrition Security for funding the wet lab processing of molecular data. The funding was awarded to Daniel Ojwang and Crispus Mbaluto through the African Biosciences Challenge Fund (ABCF) scholarship. Prof. Looney BP. from the Ecology and Evolutionary Biology Department in the University of Tennessee provided invaluable expertise in determining the authenticity of the Auricularia delicata strains from this study.

\section{References}

Bandara AR, Chen J, Karunarathna S, Hyde KD, Kakumyan P. 2015 - Auricularia thailandica sp. nov. (Auriculariaceae, Auriculariales) a widely distributed species from Southeastern Asia. Phytotaxa 208, 147-156.

Bresadola G, Hennings P, Magnus P. 1893 - Die von Herrn P. Sintenis auf der Insel Portorico 1884 1887 gesammelten Pilze. Botanische Jahrbücher für Systematik Pflanzengeschichte und Pflanzengeographie. 17, 489-501.

Chen Y-C, Eisner JD, Kattar MM, Rassoulian-Barrett SL, Lafe K, Limaye AP, Cookson BT. 2001 Polymorphic internal transcribed spacer region 1: DNA sequences identify medically important Yeasts. Journal of Clinical Microbiology 39, 4042-4051

Food Agricultural Organization 2015 - World Agriculture: Towards 2015/2030. An FAO Perspective. Edited by Bruinsma J. Earthscans Publications Limited, London.

Gardes M, Bruns, TD. 1993 - ITS primers with enhanced specificity for basidiomycetes - application to the identification of mycorrhizae and rusts. Molecular Ecology 2, 113-118.

Hussein JM, Tibuhwa DD, Mshandete AM, Kivaisi AK. 2014 - Molecular phylogeny of saprophytic wild edible mushroom species from Tanzania based on ITS and nLSU rDNA sequences. Current Research in Environmental and Applied Mycology 4(2), 250-260.

Kirk PM, Cannon PF, Minter D, Stalpers JA. 2008 - Dictionary of the Fungi. CABI Wallingford, UK.

Korabecna M, Liska V, Fajfrlik K. 2003 - Primers ITS1, ITS2 and ITS4 detect the intraspecies variability in the internal transcribed spacers and 5.8S rRNA gene region in clinical strains of fungi. Folia Microbiology 48(2), 233-238.

Looney BP, Birkebak JM, Matheny PB. 2013 - Systematics of the genus Auricularia with an emphasis on species from the southeastern United States. North American Fungi 8(6), 1-25.

Lowy B, 1951 - A morphological basis for classifying the species of Auricularia. Mycologia 43, 351358.

Lowy B, 1952 - The genus Auricularia. Mycologia 44, 656-692.

Martin P, Muruke M, Hosea K, Kivaisi AK, Zerwas N, Bauerle C. 2004 - A rapid PCR-RFLP method for monitoring genetic variation among commercial mushroom species. Biochemistry and Molecular Biology Education 32, 390-394.

Miller SL, Buyck B. 2002 - Molecular phylogeny of the genus Russula in Europe with a comparison of modern infrageneric classifications. Mycological Research 106, 259-276.

Onyango BO, Palapala VA, Arama PF, Wagai SO, Gichimu BM. 2011 - Morphological characterization of Kenyan native wood ear mushroom [Auricularia auricula (L. ex Hook.) 
Underw.] and the effect of supplemented millet and sorghum grains in spawn production. Agriculture and Biology Journal of North America 3, 2151-7517.

Palapala VA, Miheso FP, Nandi O. 2006 - Cultivation potential of indigenous species of African wood ear mushrooms. Paper presented at Masinde Muliro University, Kenya: 1-21.

Park MS, Lee H, Oh SY, Jung PE, Seok SJ, Fong JJ, Lim YW. 2014 - Species delimitation of three species within the Russula subgenus Compacta in Korea: $R$. eccentrica, $R$. nigricans, and $R$. Subnigricans. Journal of Microbiology 52(8), 631-638.

Schindel DE, Miller SE. 2005 - DNA barcoding a useful tool for taxonomists. Nature 435, 17.

Schoch CL, Seifert KA, Huhndorf S, Robert V, Spouge JL,Levesque CA. 2012 - Nuclear ribosomal internal transcribed spacer (ITS) region as a universal DNA barcode marker for Fungi. Proceedings of the National Academy of Sciences 109, 6241-6246.

Tamura KM, Kumar S. 2004 - Prospects for inferring very large phylogenies by using the neighborjoining method. Proceedings of the National Academy of Sciences (USA) 101, 11030-11035.

Tamura KM, Peterson D, Peterson N, Stecher G, Nei M, Kumar S. 2011 - MEGA6: Molecular evolutionary genetics analysis using maximum likelihood, evolutionary distance, and maximum parsimony methods. Molecular Biology and Evolution 28, 2731-2739

Tang LH, Xiao Y, Li L, Guo Q, Bian YB. 2010 - Analysis of genetic diversity among Chinese Auricularia auricula cultivars using combined ISSR and SRAP markers. Current Microbiology $61,132-40$.

Vellinga EC. 2004 - Genera in the family Agaricaceae: Evidence from nrITS and nrLSU sequences. Mycological Research 108, 354-377.

Wambua J. 2004 - Mushroom cultivation in Kenya; Mushroom growers handbook. Part III. O. U. Press p 197-203.

Weiß M, Oberwinkler F. 2001 - Phylogenetic relationships in Auriculariales and related groups hypotheses derived from nuclear ribosomal DNA sequences. Mycological Research 105, 403415.

White TJ, Bruns T, Lee S, Taylor J. 1990 - Amplification and direct sequencing of fungal ribosomal RNA genes for phylogenetics. PCR Protocols: A Guide to Methods and Applications 18, 315322. 